\title{
Indigenous Medicinal Plants as Biofilm Inhibitors for the Mitigation of Antimicrobial Resistance
}

\author{
Elikplim Kwesi Ampofo (D), ${ }^{1}$ Isaac Kingsley Amponsah ${ }^{D},{ }^{1}$ Evelyn Asante-Kwatia (D), ${ }^{1}$ \\ Francis Ackah Armah $\mathbb{D}^{2}{ }^{2}$ Philip Kobla Atchoglo, ${ }^{1}$ and Abraham Yeboah Mensah (D) \\ ${ }^{1}$ Department of Pharmacognosy, Faculty of Pharmacy and Pharmaceutical Sciences, College of Health Sciences, \\ Kwame Nkrumah University of Science and Technology, Kumasi, Ghana \\ ${ }^{2}$ Department of Biomedical Sciences, School of Allied Health Sciences, University of Cape Coast, Cape Coast, Ghana
}

Correspondence should be addressed to Evelyn Asante-Kwatia; eamireku@knust.edu.gh

Received 28 April 2020; Revised 8 October 2020; Accepted 14 October 2020; Published 24 October 2020

Academic Editor: Ergin Murat Altuner

Copyright (C) 2020 Elikplim Kwesi Ampofo et al. This is an open access article distributed under the Creative Commons Attribution License, which permits unrestricted use, distribution, and reproduction in any medium, provided the original work is properly cited.

\begin{abstract}
The majority of indigenes in the rural areas of Ghana use herbal medicines for their primary health care. In this study, an ethnobotanical survey was undertaken to document medicinal plants used by traditional healers in the Ejisu-Juaben district in the Ashanti region of Ghana to treat infections and to further investigate the antibiofilm formation properties of selected plants in resisting pathogenic bacteria. Seventy medicinal plants used by traditional practitioners for the treatment of skin infections and wounds were documented from the ethnobotanical survey. Forty out of the seventy plants were collected and their methanol extracts evaluated for antimicrobial activity by the agar diffusion assay. Extracts that showed antibacterial activity were tested for biofilm inhibitory activity, and the most active plant was subsequently purified to obtain the active constituents. Biofilm formation was significantly mitigated by petroleum ether, ethyl acetate, and methanol extracts of Holarrhena floribunda stem bark. Bioassayguided fractionation of an alkaloidal extract prepared from the methanol fraction led to the isolation of three steroidal alkaloids, namely, holonamine, holadienine, and conessine. The isolated compounds demonstrated varying degrees of biofilm formation inhibitory properties. The current study reveals that screening of indigenous medicinal plants could unravel potential leads to salvage the declining efficacy of conventional antibiotics. Holarrhena floribunda stem bark extract has strong biofilm formation inhibition properties, which could be attributed to the presence of steroidal alkaloids.
\end{abstract}

\section{Introduction}

Antimicrobial resistance (AMR) has become a major public health concern worldwide as it reduces the effectiveness of antibiotic therapies and increases morbidity, mortality, and health care costs [1]. Current evidence suggests that freefloating bacteria attach onto solid surfaces and create a complex polysaccharide matrix called the biofilm, which protects them from antimicrobial agents [2,3]. Biofilms have great significance for public health because biofilm-associated microorganisms exhibit dramatically high resistance to antimicrobial agents than planktonic forms [4]. This is because in the biofilm state multiple bacterial species form a polymicrobial community with several advantages including a more efficient DNA sharing and quorum sensing system, passive resistance, and metabolic cooperation, which protects them from antimicrobial agents and host immune responses [5].

Some extracts of neotropical rainforest plants have shown remarkable biofilm inhibitory effects $[1,6]$. Triterpenes with inhibitory effects against $P$. aeruginosa biofilms were isolated from Diospyros dendo [7]. The antibiofilm activity of Azadirachta indica extract was employed to mitigate microbiologically influenced corrosion in underground pipe lines as an environmentally benign way of managing corrosion [8]. This put natural products with antibiofilm activity in the spotlight for a wide range of applications to address health and industrial issues. In the 
rural and peri-urban areas like the Ejisu-Juaben district in the Ashanti region of Ghana, access to medical care is limited, and most indigenes rely on herbal medicine practitioners for their primary health needs. Herbal medicines are highly valued and enjoy patronage by a large number of the populace due to its acceptability, affordability, and perceived safety [9]. In this study, an ethnobotanical survey was undertaken in the Ejisu-Juaben district to obtain comprehensive data on plant-based remedies commonly used by traditional healers to treat infection. Furthermore, the biofilm formation inhibitory activities of selected plant extracts were investigated and some bioactive constituents identified.

\section{Materials and Methods}

2.1. Chemicals. Chemicals used in the assay were purchased from Sigma and Aldrich chemicals, UK, and Ciprofloxacin was purchased from DENK PHARMA GmbH \& Co, Germany. All organic solvents were of analytical grade and obtained from BDH, Laboratory Supplies (Merck Ltd, Lutterworth, UK).

2.2. Study Area. This ethnobotanical study was conducted in the Ashanti Region of Ghana, specifically in the Ejisu-Juaben district (Figure 1). The Ejisu-Juaben district is one of the 27 administrative and political districts in the Ashanti Region of Ghana. It is located in the central part of the region $\left(1.15^{\circ} \mathrm{N}\right.$ and $1.45^{\circ} \mathrm{N}$ and longitude $6.15^{\circ} \mathrm{W}$ and $7.00^{\circ} \mathrm{W}$ ) and has an estimated population of about 150,000 with about $72 \%$ residing in the rural areas (Ghana Statistical Service, 2014). The district lies within a semi-deciduous forest zone where flora and fauna is diverse and composed of different species of both economic and ornamental importance. The communities are well known for their traditional beliefs and the use of plants for medicine (Ghana Statistical Service, 2014).

2.3. Data Collection. The ethnobotanical survey was carried out in May, 2016, with the help of some members of the Ghana National Association of Traditional Healers, Ashanti region (GNATH), a subunit of the Ghana federation of traditional healers associations (GHAFTRAM). The research was conducted in five villages in the district, namely, Bonwire, Ejisu, Onwe, Besease, and Juaben. Participants were informed of the objectives of the survey and personal visits were made to their homes and practice sites. A prior informed consent form (translated into the local dialect) was given to the participants to sign after the objectives of the study had been explained. In keeping with the traditional customs, appropriate gifts and drinks were given to the participants for the time and the courtesies extended to us. Data were collected through a survey employing openended, semi-structured questionnaires in English and translated into the local dialect (Ashanti-Twi). Thirty traditional healers participated in the survey. All participants provided information on the methods of practice, vernacular names of anti-infective plant remedies employed by them, methods of preparation, and administration of these remedies. Ethical approval for the study was obtained from the chiefs of the studied areas and the GNATH.

2.4. Plant Material Collection. The interviews were followed by the collection of plants under the supervision of three traditional healers. Seventy medicinal plants were documented from the survey (Supplementary Material-Table S1). Forty of these, cited more than five times by $20 \%$ of the traditional healers, were collected from the Ejisu-Juaben district for further investigation. They were authenticated by Dr. George Henry Sam of the Department of Herbal Medicine. Voucher specimens of all the plants have been deposited in the Herbarium of the Herbal Medicine Department, Faculty of Pharmacy \& Pharmaceutical Sciences, KNUST, with defined ID numbers. The names and the authenticity of the plant species were confirmed using the Electronic Plant Information Centre (ePIC, http://epic.kew. org/).

2.4.1. Preparation of Plant Extracts for Antibacterial Susceptibility Test. One hundred and fifty grams each of the forty selected plants were dried in the shade for 7 days, milled into a coarse powder using a warring blender, and extracted with $80 \%$ methanol by cold maceration. They were filtered, concentrated on a rotary evaporator, and dried on a water bath. They were then stored in a cool dry place until required for use.

2.4.2. Fractionation of $H$. floribunda Extract and Preparation of Alkaloidal Fraction. Coarsely powdered stem bark of H. floribunda $(3.49 \mathrm{~kg})$ was Soxhlet-extracted successively using $3 \mathrm{~L}$ each of petroleum ether (pet-ether), ethyl acetate (EtOAc), and methanol $(\mathrm{MeOH})$ for 48 hours. The extracts obtained were concentrated on a rotary evaporator under low temperature and further dried to afford the pet-ether (PHE-5.2\%), ethyl acetate extract (EHE-19.85\% w/w), and a methanol extract (MHE-16.15\% w/w). An alkaloidal fraction was obtained by dissolving $156.9 \mathrm{~g}$ of MHE in $1.25 \mathrm{~L}$ of $10 \%$ acetic acid. The resulting solution was made distinctly alkaline with $70 \mathrm{~mL}$ of strong ammonia. The alkaloids were then extracted with $2.5 \mathrm{~L}$ of chloroform. The chloroform extract was concentrated by evaporation on a rotary evaporator to yield $52.4 \mathrm{~g}$ of crude alkaloidal fraction.

\subsection{Antimicrobial Assay}

2.5.1. Microorganisms. Resistant clinical strains of Staphylococcus aureus ATCC 25923 (A), Streptococcus pyogenes clinical strain (B), Escherichia coli ATCC 25922 (C), Pseudomonas aeruginosa ATCC 27853 (D), and Klebsiella pneumonia clinical strain (E) were obtained from the Department of Pharmaceutical Microbiology, Faculty of Pharmacy and Pharmaceutical Sciences, KNUST, and used for the study. They were authenticated according to their respective features on selective media, maintained in 30\% glycerol broth and stored at $-40^{\circ} \mathrm{C}$ in a frost-free freezer. The strains were aseptically subcultured into sterile Mueller 


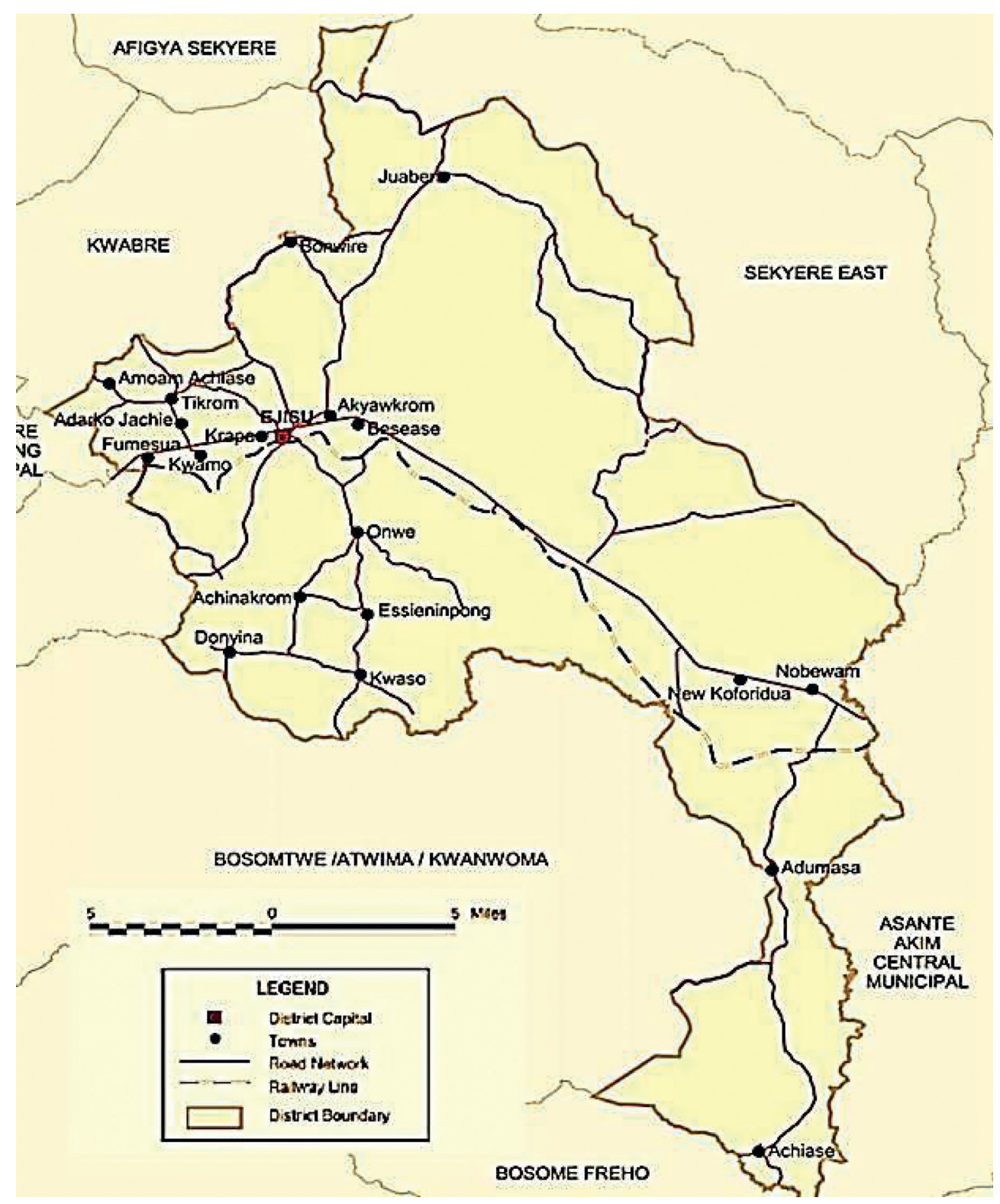

Figure 1: Map of Ejisu-Juaben district (Source: Ghana Statistical Service).

Hinton broth and incubated at $37^{\circ} \mathrm{C}$ for 24 hours prior to use. For all assays, the working culture was prepared by standard dilution in distilled water and the bacterial suspension was adjusted to a turbidity matching that of $0.5 \mathrm{McFarland}$ Standard (containing $\sim 10^{8}$ colony forming units $(\mathrm{CFU}) / \mathrm{mL}$ ) according to the CLSI guidelines $[10,11]$. To ensure cellular quiescence, this dilution was maintained at $4^{\circ} \mathrm{C}$.

2.5.2. Antimicrobial Susceptibility Test. The agar well diffusion method as described by Jorgenson et al. was used, and the method description partly reproduces their wording [12]. Forty medicinal plants cited more than five times by $20 \%$ of the traditional healers (Percentage of respondents with the knowledge about the use of a particular plant) were investigated for antibacterial activity (shown by asterisk '*' on Table S1, Supplementary Material). Briefly, sterile molten Mueller Hinton agar (20 mL each) was seeded with $100 \mu \mathrm{L}$ of standardized test organism and poured into $90 \mathrm{~mm}$ sterile Petri dishes. After solidification, equivalent wells were drilled in each of the petri dishes using a sterile cork borer (No. 5) and filled with the extracts at concentrations of $5 \mathrm{mg} /$ $\mathrm{mL}$. The petri dishes were allowed to stand for 1 hour at $25^{\circ} \mathrm{C}$ to allow for diffusion of extracts into the seeded agar and then incubated at $37^{\circ} \mathrm{C}$ for 24 hours. Zones of growth inhibition were determined after incubation. Ciprofloxacin was used as the reference drug. The experiment was performed in triplicate, and the results are presented as the mean of three values \pm the standard deviation.

2.5.3. Analysis of Biofilm Production by Test Organisms. To qualitatively investigate the biofilm-producing abilities of the selected bacteria, the method described by Amaral et al. 
was employed with some modification. Briefly, a sterile glass slide placed in a sterile petri dish was overlaid with $20 \mathrm{~mL}$ of Mueller Hinton broth seeded with $100 \mu \mathrm{L}$ of standardized culture $\left(\sim 10^{8} \mathrm{CFU} / \mathrm{mL}\right)$. After 24 and 48 hours of incubation, the slides were washed with normal saline to remove the floating planktonic bacteria, and the biofilm was fixed by heating the slide. Slides were then stained with $0.1 \%$ crystal violet for 15 minutes, washed with distilled water, air dried, and observed under the microscope. Photomicrographs of bacterial biofilms were recorded at a magnification of $\times 100$ [13].

\subsubsection{Quantitative Determination of the Biofilm Formation} by the Organisms. Quantitative assessment of biofilm formation was done using the Crystal Violet (CV) assay as described by Sasirekha et al. and the description partly reproduces their wording [6]. Briefly, fresh Mueller Hinton broth $(100 \mu \mathrm{L})$ was seeded with $10 \mu \mathrm{L}$ of standardized microorganism culture in a 96-well microtiter plate and incubated at $37^{\circ} \mathrm{C}$ for 24 hours. After incubation, the planktonic cells were aspirated and the wells were washed with sterile water to remove the free-floating bacteria. The adhering biofilm was fixed by heating and then stained with $0.1 \%$ crystal violet. The wells were then washed with sterile water and dried. The amount of biofilm formed was quantified by adding $200 \mu \mathrm{L}$ of $95 \%$ ethanol/acetic acid mixture (1:1) and the optical density (OD) of the resulting solution was measured at $595 \mathrm{~nm}$. Background absorbance was compensated for by subtracting the optical density (OD) of sterile broth (negative control). The experiment was carried out in triplicate and the mean OD was considered. A cutoff OD was determined from the negative control and used to separate nonbiofilm from biofilm-producing microorganisms following standard methods [11].

2.5.5. Biofilm Inhibition Activity. Eight plant extracts that showed antimicrobial activity in the susceptibility test were investigated in this assay. The test was carried out in 96-well microtiter plates. Briefly, $100 \mu \mathrm{L}$ of sterile liquid broth medium was inoculated with $10 \mu \mathrm{L}$ of standardized overnight culture of the selected bacterium, and volumes of plant extracts reconstituted in distilled water were added to obtain concentrations of $10,5,2.5$, and $1.25 \mathrm{mg} /$ $\mathrm{mL}$. For the determination of the minimum biofilm inhibitory concentrations of fractions and pure compounds, a concentration range between 1 and $0.0078 \mathrm{mg} / \mathrm{mL}$ was used. Ciprofloxacin was used as the reference drug. Plates were incubated at $37^{\circ} \mathrm{C}$ for 24 hours. The experiment was performed in triplicate. After the incubation, the liquid suspension was removed and the amount of biofilm formed determined as described in Section 2.5.4.

2.6. Data Management and Analysis. The survey data were analyzed using the Statistical Package for Social Sciences (SPSS) version 22.0 for windows and Graph Pad Prism (Version 5 for windows, San Diego, USA).
2.7. Chromatographic Fractionation of Alkaloid Extract. The alkaloid extract $(50 \mathrm{~g})$ was loaded onto a glass column $(60 \mathrm{~cm} \times 3 \mathrm{~cm})$ packed with silica gel $(70-230 \mathrm{mesh})$. It was then eluted with gradient mixtures of pet-ether, EtOAc, and $\mathrm{MeOH}$. Fifty-six fractions were collected in $60 \mathrm{~mL}$ aliquots and bulked together into six subfractions based on their TLC profiles coded CF 1-6. Fraction CF1 (9.5 g) was further purified by column chromatography to obtain sixty-three fractions bulked into six subfractions, which were labeled HF1, HF2, HF3, HF4, HF5, and HF6. Fraction HF1 (1.19 g) was subjected to purification by preparative HPLC $\left[\mathrm{MeOH}-\mathrm{H}_{2} \mathrm{O}+0.1 \% \mathrm{HCOOH} 98: 2,2 \mathrm{~mL} \mathrm{~min}^{-1}\right]$ to yield pure compounds, namely, HF1A, HF1B, and HF1C. Details of HPLC experimental procedures are provided in supplementary data (available here).

\section{Results}

3.1. Sociodemographic Details of Respondents. All the traditional healers interviewed belonged to the Akan tribe. The inclusion criterion for the survey was that the practitioner used herbal medicine. They comprised $43 \%$ men and 57\% women aged between 21 and 80 years. The categories of practitioners included diviners (17\%), herbalists (67\%), clairvoyants (3\%), fetish priests (10\%), and bone setters (3\%). A majority of respondents (61\%) had practiced for more than 15 years. Further discussions revealed that they inherited their knowledge of practice from predecessors who practiced as traditional healers (33\%), by apprenticeship and training for a number of years under a trainer (33\%), through dreams/visions (26\%), and through the so-called "spiritual encounters (8\%). A majority of respondents (77\%) indicated that they harvested their plants from the wild (including collections from a nearby arboretum in the district, Bobiri Forest Reserve) and the rest they cultivated in their farms and backyards (23\%).

\subsection{Plants Used for the Treatment of Infections in the Ejisu-} Juaben District. Seventy plants used by the traditional practitioners for the treatment of skin, wounds, and other infections were documented from the survey (Supplementary Material-Table S1). The plants were distributed across 63 genera belonging to 31 families, mostly Fabaceae (9), Euphorbiaceae (6), Moraceae (6), and Meliaceae (6). The vernacular names of plants, uses, methods of preparation, and the administration of remedies were documented.

3.3. Antimicrobial Activity of Plant Extracts. Forty medicinal plants cited more than five times by $20 \%$ of the traditional healers were investigated for antibacterial activity by the agar diffusion assay. Eight out of the forty plants exhibited antimicrobial activities as determined by the zones of inhibition of susceptible microbes (Table 1). These were Bridelia stenocarpa (MBS), Anogeissus sericea (MAS), Trichilia lanata (MTL), Grossera vignei (MGV), Acacia ataxacantha (MAA), Albizia ferruginea (MAF), Holarrhena floribunda (MHF), and Triplochiton scleroxylon (MTS) (Figure 2). 
TABLE 1: Mean zones of inhibition of extracts in agar well diffusion assay.

\begin{tabular}{|c|c|c|c|c|c|c|c|c|c|}
\hline \multirow{2}{*}{ Microorganism } & \multicolumn{9}{|c|}{ Mean zones of inhibition $(\mathrm{mm}) \pm \mathrm{SD}$} \\
\hline & MBS & MAS & MTL & MGV & MAA & MAF & MHF & MTS & CIP \\
\hline \multirow{2}{*}{ S. aureus } & 11.7 & 12.7 & 11.3 & 11.7 & 21.7 & 12.3 & 14.7 & 16.3 & 26.7 \\
\hline & \pm 0.7 & \pm 2.1 & \pm 1.8 & \pm 1.2 & \pm 2.9 & \pm 3.4 & \pm 2.4 & \pm 3.1 & \pm 1.7 \\
\hline \multirow{2}{*}{ K. pneumoniae } & 8.3 & 10.7 & 5.7 & 8.3 & 12.3 & 8.7 & 12.3 & 14.3 & 28.7 \\
\hline & \pm 0.9 & \pm 2.1 & \pm 0.6 & \pm 2.1 & \pm 3.1 & \pm 1.1 & \pm 1.9 & \pm 1.5 & \pm 0.8 \\
\hline \multirow{2}{*}{ S. pyogenes } & 12.7 & 9.3 & 12.7 & 11.7 & 20.7 & 10.3 & 11.3 & 10.7 & 18.3 \\
\hline & \pm 1.9 & \pm 1.6 & \pm 2.3 & \pm 0.8 & \pm 4.3 & \pm 2.6 & \pm 2.0 & \pm 2.2 & \pm 1.7 \\
\hline \multirow{2}{*}{ E. coli } & 13.7 & 7.7 & 4.3 & 16.7 & 14.7 & 9.7 & 15.7 & 9.3 & 19.7 \\
\hline & \pm 4.2 & \pm 0.5 & \pm 0.8 & \pm 4.1 & \pm 1.8 & \pm 4.1 & \pm 0.9 & \pm 3.3 & \pm 0.9 \\
\hline \multirow{2}{*}{ P. aeruginosa } & 12.3 & 12.7 & 13.7 & 14.3 & 10.3 & 11.3 & 16.7 & 5.7 & 10.3 \\
\hline & \pm 1.7 & \pm 2.8 & \pm 0.9 & \pm 2.3 & \pm 1.2 & \pm 3.7 & \pm 0.4 & \pm 2.2 & \pm 0.6 \\
\hline
\end{tabular}

MBS, Bridelia stenocarpa; MAS, Anogeissus sericea; MTL, Trichilia lanata; MGV, Grossera vignei; MAA, Acacia ataxacantha; MAF, Albizia ferruginea; MHF, Holarrhena floribunda MTS, Triplochiton scleroxylon; CIP, ciprofloxacin; SD, standard deviation.

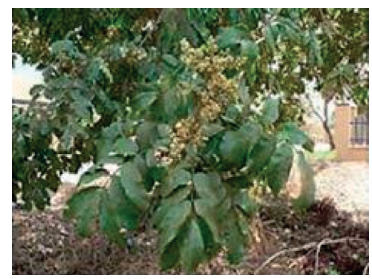

(a)

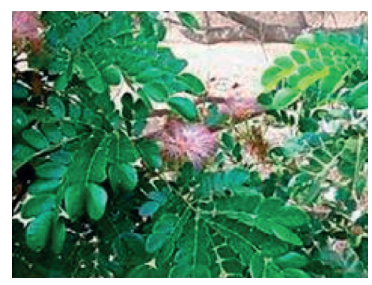

(e)

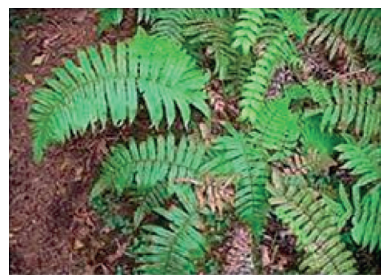

(b)

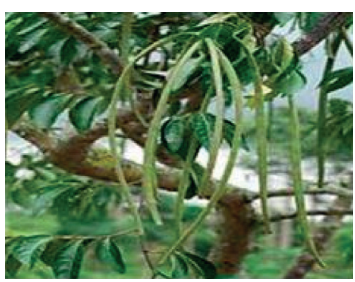

(f)

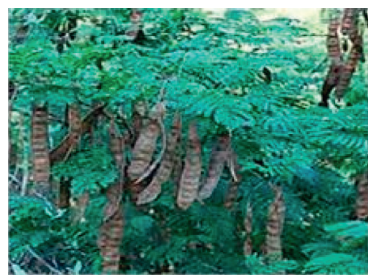

(c)

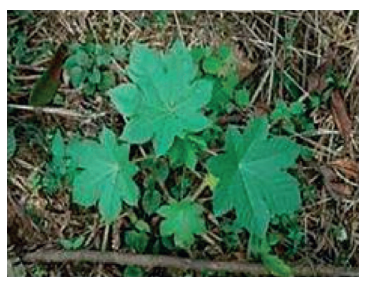

(g)

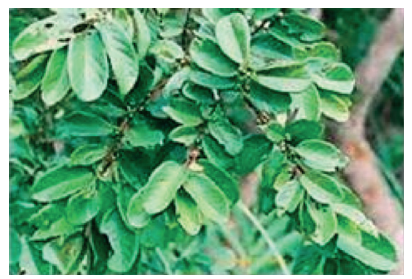

(d)

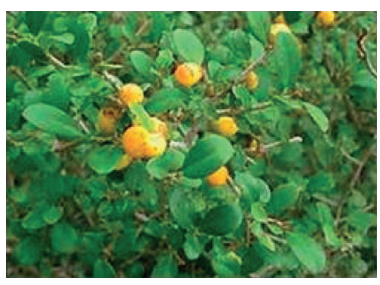

(h)

FIGURe 2: : Selected plants for biofilm formation inhibitory activity. (a) Trichilia lanata (MTL). (b) Grossera vignei (MGV). (c) Acacia ataxacantha (MAA). (d) Bridelia stenocarpa (MBS). (e) Albizia ferruginea (MAF). (f) Holarrhena floribunda (MHF). (g) Triplochiton scleroxylon (MTS). (h) Anogeissus sericea (MAS).

3.4. Biofilm Formation Ability of Selected Microorganisms. All the clinical strains selected for the assay had the ability to produce biofilms. This was first determined qualitatively from photomicrographs of glass slides with adhering bacterial biofilms stained with $0.1 \%$ crystal violet (Figure 3 ). To quantify the amount of the biofilm produced, the optical densities (OD) of reconstituted biofilm solutions from the microtiter plate assay were determined (Table 2 ). A negative control was included and used to calculate a cutoff OD value, which separates a nonbiofilm producer from a biofilmproducing organism according to the established standards $[11,14]$. From calculations, the following parameters were set: $\mathrm{OD}<0.12$ as weak biofilm producers; OD between 0.12 and 0.24 as moderate biofilm producers; and OD $>0.24$ as high biofilm producers. The rank order of biofilm-producing ability was $S$. aureus $>S$. pyogenes $>E$. coli $>P$. aeruginosa $>$ K. pneumoniae. S. aureus was therefore selected for the investigation of biofilm formation inhibitory property of H. floribunda.
3.5. Biofilm Formation Inhibitory Effect of Plant Extracts. The methanol extracts of the eight plants that showed antibacterial activity were further tested for biofilm inhibitory activity against $S$. aureus. The ability of the extracts to considerably inhibit biofilm formation by a strong biofilm producer (i.e., S. aureus) was determined and the minimum biofilm inhibitory concentration (MBIC) recorded. From the experiments, six out of the eight plant extracts failed to significantly inhibit biofilm formation by $S$. aureus at concentrations up to $10 \mathrm{mg} / \mathrm{mL}$ (Figure 4(a)-4(b)). Meanwhile, the methanol extracts of two plants, Grossera vignei (MGV) and Holarrhena floribunda (MHF), showed appreciable biofilm formation inhibitory activities. At a concentration of $1.25 \mathrm{mg} / \mathrm{mL}$, MHF reduced S. aureus from a high biofilm producer $(\mathrm{OD}>1.38)$ to a low biofilm-producing organism with an $\mathrm{OD}<0.24$ (Figure 4(b)). G. vignei, methanol extract $(\mathrm{MGV})$ on the other hand, reduced the biofilm production of $S$. aureus to a moderate biofilm producer at concentrations of 5 to $10 \mathrm{mg} / \mathrm{mL}$ (Figure $4(\mathrm{~b})$ ). 


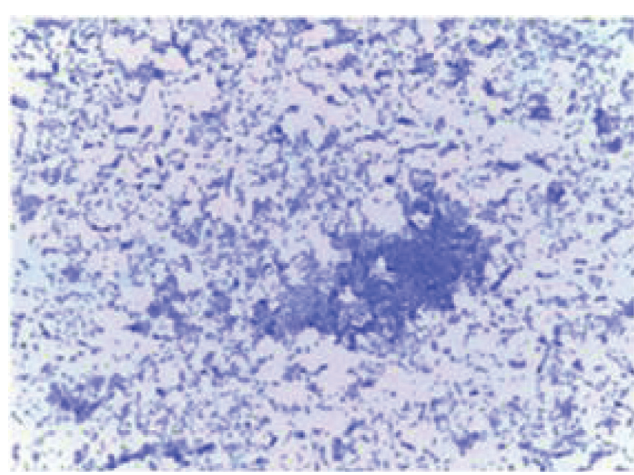

(a)

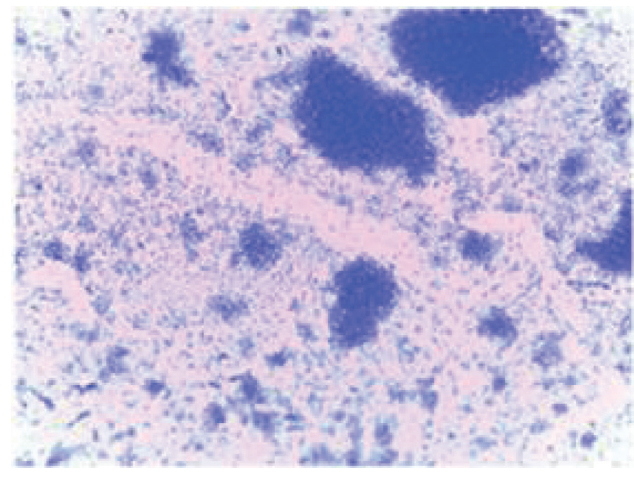

(c)

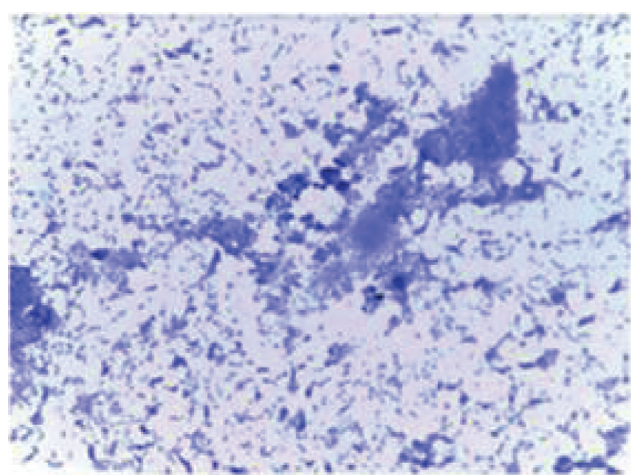

(b)

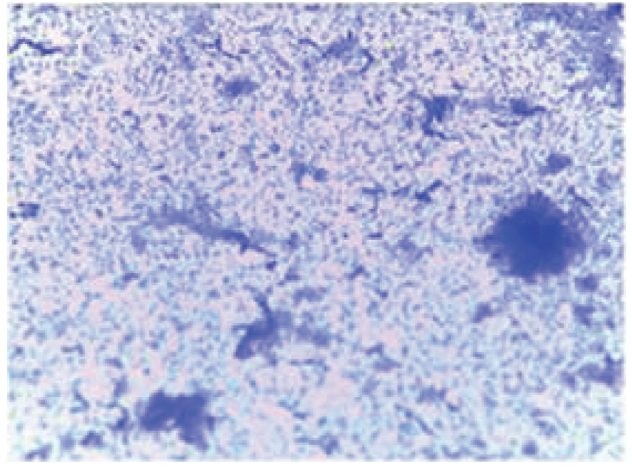

(d)

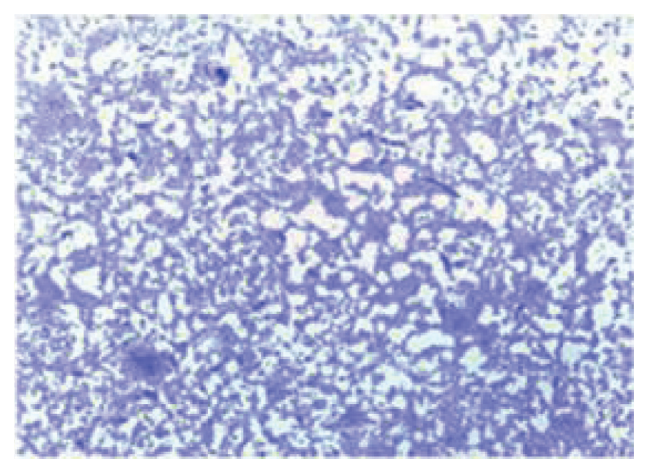

(e)

Figure 3: Photomicrographs of adhering bacterial cells (biofilms) stained with $0.1 \%$ crystal violet on a glass slide after 24 hours, observed at $\times 100$ : (a) S. aureus. (b) S. pyogenes. (c) E. coli. (d) P. aeruginosa. (e) K. pneumoniae.

TABLE 2: Optical densities of biofilms produced by various microorganisms.

\begin{tabular}{lcccccc}
\hline Organism & S. aureus & S. pyogenes & E. coli & P. aeruginosa & K. pneumoniae & Control \\
\hline OD & $1.38 \pm 0.049$ & $1.37 \pm 0.083$ & $1.25 \pm 0.034$ & $1.15 \pm 0.103$ & $0.86 \pm 0.044$ & $0.05 \pm 0.004$ \\
\hline
\end{tabular}

OD, optical density.

3.6. Biofilm Formation Inhibitory Effect of $H$. floribunda Solvent Fractions and Pure Compounds. H. floribunda crude extract (MHF), which showed the highest biofilm formation inhibition toward S. aureus (Figure 4(a)), was successively fractionated to obtain the pet-ether (PHE), ethyl acetate (EHE), and methanol (MHE) fractions, and their biofilm inhibitory effects were investigated. MHE showed the highest biofilm formation inhibitory effect with an MBIC of
$0.0625 \mathrm{mg} / \mathrm{mL}$ (Table 3). An alkaloidal fraction (AKL) was further prepared from MHE and purified by column and high-performance liquid chromatography to yield the compounds HF1A, HF1B, and HF1C (Figure 5). The amount of biofilm formed recorded as optical density in the presence of different concentrations of PHE, EHE, MHE, $\mathrm{AKL}$ and the isolated compounds toward $S$. aureus is demonstrated in Figure 6(a)-6(b). Among the isolated 


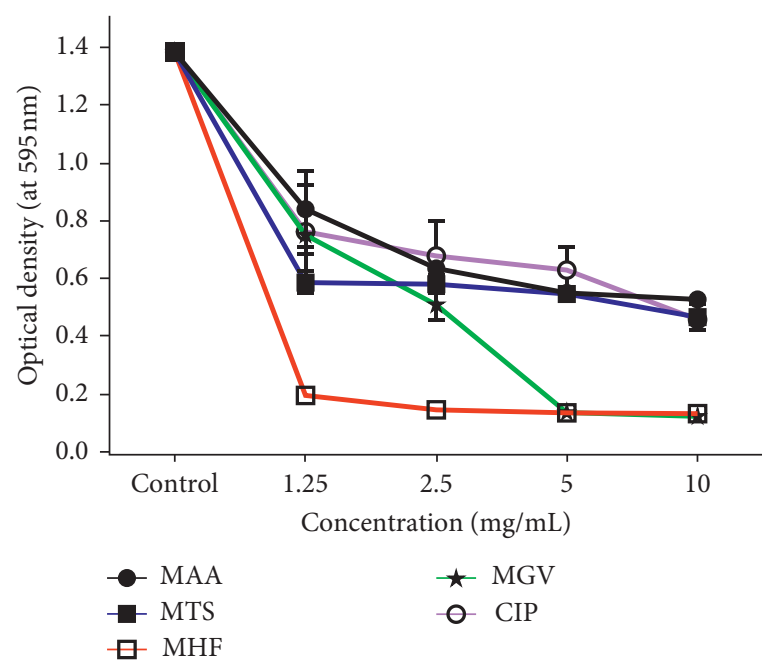

(a)

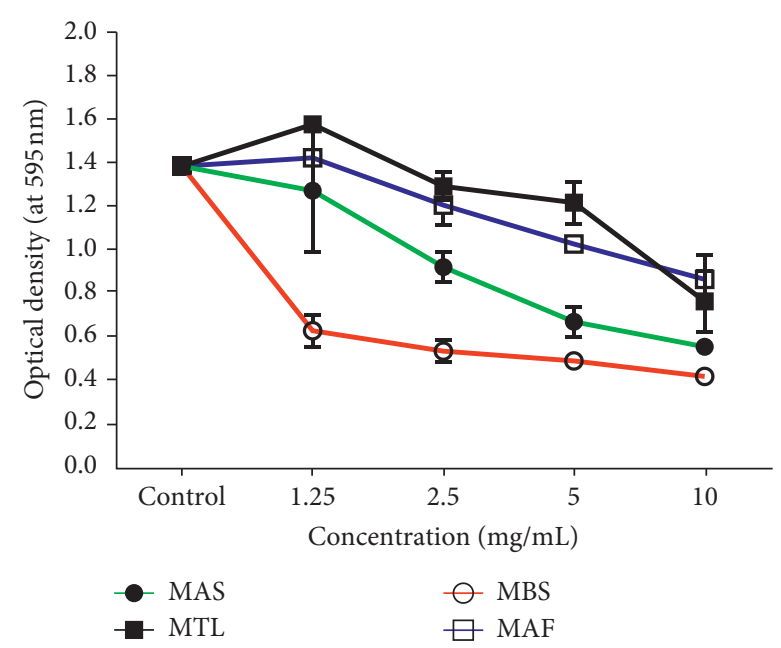

(b)

Figure 4: (a-b) Graphs showing the effect of different concentrations of extracts on the amount of biofilm formed (optical density-OD) by S. aureus. MBS, Bridelia stenocarpa; MAS, Anogeissus sericea; MTL, Trichilia lanata; MGV, Grossera vignei; MAA, Acacia ataxacantha; MAF, Albizia ferruginea; MHF, Holarrhena floribunda; MTS, Triplochiton scleroxylon; CIP, ciprofloxacin.

TABLE 3: Minimum biofilm inhibitory concentrations of fractions, isolated compounds HF1A, HF1B, and HF1C, and the reference drug against $S$. aureus.

\begin{tabular}{lc}
\hline Extracts/compounds & Minimum biofilm inhibitory concentration (MBIC) (mg/mL) \\
\hline MHF & 1.00 \\
PHE & $>1.00$ \\
MHE & 0.0625 \\
EHE & $>1.00$ \\
AKL & 0.125 \\
HF1A & 0.250 \\
HF1B & $>1.00$ \\
HF1C & 0.50 \\
CIP & 0.50 \\
\hline
\end{tabular}

CIP: ciprofloxacin; MHF: crude extract; MHE: methanol fraction; EHE: ethyl acetate fraction; PHE: pet-ether fraction; AKL: alkaloidal fraction. HF1A, HF1B, and HF1C are isolated compounds.

compounds, HF1A had the highest activity with an MBIC of $0.25 \mathrm{mg} / \mathrm{mL}$ toward $S$. aureus.

3.7. Characterization of the Compounds. Compounds HF1A, $\mathrm{HF} 1 \mathrm{~B}$, and HF1C (Figure 6) were identified based on comparison of mass spectral, ${ }^{1} \mathrm{H}$ and ${ }^{13} \mathrm{C} \mathrm{NMR}$ data to published literature as holonamine, holadienine, and conessine, respectively $[15,16]$.

\section{Discussion}

A number of researches have documented the utilization of herbal medicines in some Ghanaian communities. One of these communities is based in the Ejisu-Juaben district where the use of herbal medicine is widespread and highly diverse due to the floristic and cultural diversity of various communities [17]. In this study, medicinal plants traditionally used for the treatment of infections in the EjisuJuaben district have been documented through an ethnobotanical survey. Documentation of such useful medicinal plants is important to facilitate future research on the efficacy of these plants and allow for the appropriate incorporation of the most effective herbal remedies into primary health care.

Further in this study, the biofilm formation inhibitory effect of selected medicinal plants against biofilm-producing Staphylococcus aureus was investigated. S. aureus biofilms are known to typically colonize both artificial and tissue surfaces in humans and cause serious infections including implant-associated infections, cystic fibrosis, lung infections, and chronic wounds [18]. Among the tested plants, Holarrhena floribunda stem bark exhibited the highest biofilm formation inhibitory activity against $S$. aureus. Three extracts, pet-ether (PHE), ethyl acetate (EHE), and methanol (MHE) fractions, were prepared from the crude extract and tested for biofilm inhibitory activity. The methanol fraction exhibited the highest antibiofilm activity among the fractions, extracts, and the reference control. Crude alkaloids (AKL) precipitated from this methanol fraction also showed considerable activity although twofold less active than the 
<smiles>C[C@H]1N=C[C@]23C[C@@H](O)[C@@H]4[C@@H](CCC5=CC(=O)C=C[C@]54C)[C@H]2CC[C@H]13</smiles>

(a)

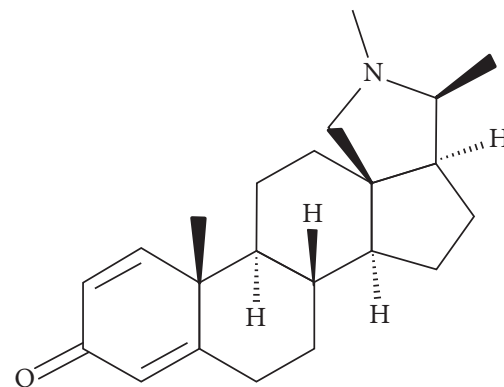

(b)

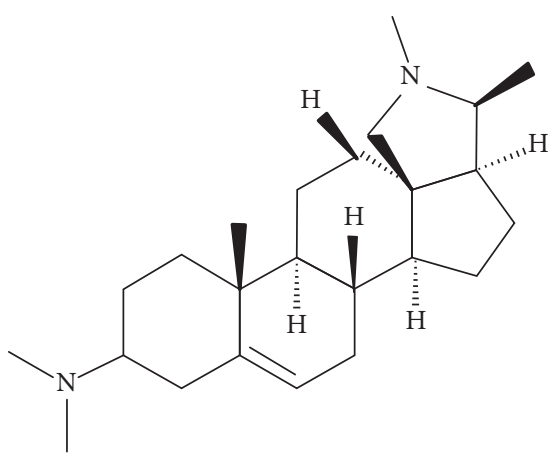

(c)

Figure 5: Steroidal alkaloids from the stem bark of $H$. floribunda. (a) Holonamine. (b) Holadienine. (c) Conessine.

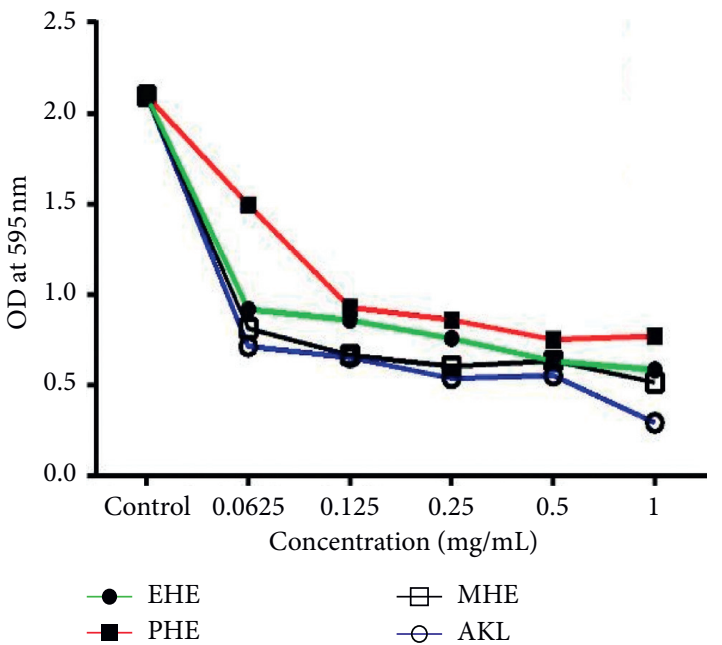

(a)

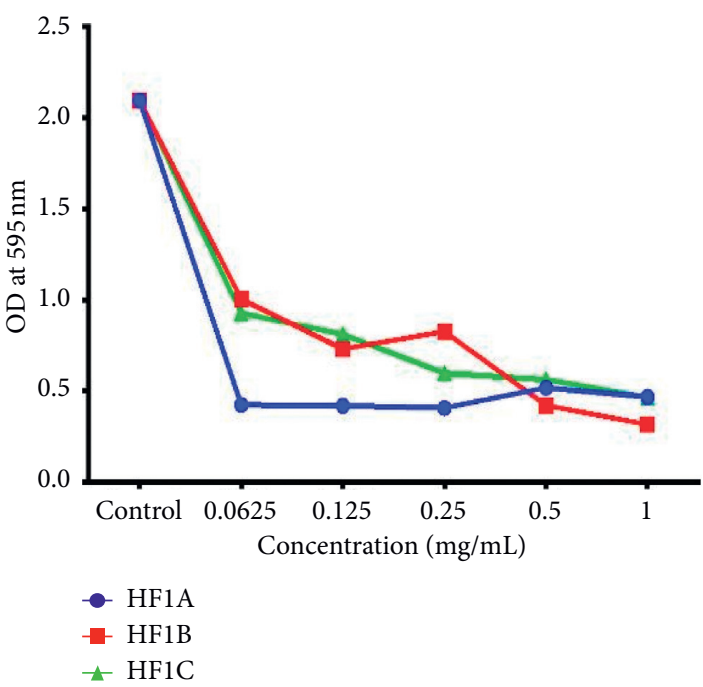

(b)

FiguRE: 6 (a-b) Graphs showing the effect of different concentrations of fractions and isolated compounds on the amount of biofilm formed (optical density-OD) by S. aureus. EHE: EtOAc fraction; PHE: pet-ether fraction; MHE: MeOH fraction; AKL: alkaloidal fraction; HF1A: holonamine; HF1B: holadienine; HF1C: conessine.

methanol fraction (Table 3). Bioassay-guided fractionation of the bioactive alkaloidal fraction revealed the presence of the steroidal alkaloids, holonamine (HF1A), holadienine (HF1B), and conessine (HF1C), which also showed considerable antibiofilm forming activity. These compounds have been previously reported from $H$. floribunda and other Holarrhena species $[15,16,19]$. This is however the first report of the biofilm formation inhibitory effect of $H$. floribunda and the isolated plant constituents. Thus, fractionation of the stem bark extract of $H$. floribunda, to some extent, afforded much potent antibiofilm methanol fraction, which owed its activity, in part, to its constituent alkaloids.

Holarrhena floribunda is well known in African traditional medicine for the treatment of malaria, diarrhea, dysentery, diabetes, skin diseases, swellings, and urinary and sexually transmitted infections $[20,21]$. The antibacterial activity demonstrated by the crude extract and the solvent fractions of the $H$. floribunda stem bark in the current work is consistent with previous reports on the same plant [22-26]. Steroidal alkaloids are the major types of phytoconstituents identified in $H$. floribunda $[16,27,28]$. Flavonoids, fatty acid esters, and trichothecenes have also been reported [29-31]. The antibacterial activity of Holarrhena species has been attributed to the presence of steroidal alkaloids [22,32-34]. Conessine (HF1C) is a well-established constituent of $H$. floribunda [19]. Although the antibiofilm inhibitory property of $H$. floribunda and its constituents was not previously investigated, a recent study demonstrated the resistance-modifying ability of conessine through the inhibition of multidrug efflux pumps [35-38]. Another study has shown that compounds with efflux pump inhibition may also cause biofilm disruption [39]. This assertion makes the current results of the antibiofilm formation effect of conessine very plausible. The activity of the individual isolated compounds was lower than the crude alkaloid fraction, suggesting the possible role of synergism in the activity of the steroidal alkaloids. This is consistent with a previous study 
by Li-Na et al. [40], which reported synergistic antibacterial activity of steroidal alkaloids isolated from Holarrhena antidysenterica against methicillin-resistant Staphylococcus aureus. In a previous study, the hydroalcoholic extract $H$. antidysenterica inhibited the formation of biofilm in opportunistic pathogenic Salmonella typhimurium [41]. This puts Holarrhena species in the spotlight as potential prospects of antibacterial agents with biofilm inhibitory activity.

\section{Conclusion}

This work has provided comprehensive data on the medicinal plants used in the treatment of infection in Ghana. These data can serve as evidential support for the clinical development of a number of medicinal plant remedies as adjuvant therapy. The biofilm formation inhibitory effect of Holarrhena floribunda and its constituents validates the ethnomedicinal use of the plant in infectious disease treatment and points to the possible presence of other potentially effective antibacterial constituents in the plant.

\section{Data Availability}

The raw data/results from experiments used to arrive at the findings of this study are available from the corresponding author upon request. Previous reports that were used to support this study are cited at relevant places within the text as references.

\section{Conflicts of Interest}

The authors have no conflicts of interest to declare.

\section{Acknowledgments}

The authors are grateful to Mr. Clifford Asare of the Herbal Medicine Department of Faculty of Pharmacy and Pharmaceutical Sciences, KNUST, and the curator of the Herbarium of the Botany Department, University of Ghana. The authors appreciate the support and assistance of the Ghana National Association of Traditional Healers Ashanti region (GNATH) for their assistance. This work was supported by the Building Stronger Universities (BSU) Grant, WP3.

\section{Supplementary Materials}

Methods: general experimental procedures. Liquid chromatography-mass spectrometry (LC-MS). Results: Table S1: medicinal plants used for the management of skin and wound infections as well as other infective conditions in the Ejisu-Juaben district of Ghana. (Supplementary Materials)

\section{References}

[1] C. M. Romero, C. G. Vivacqua, M. B. Abdulhamid et al., "Biofilm inhibition activity of traditional medicinal plants from Northwestern Argentina against native pathogen and environmental microorganisms," Revista da Sociedade Brasileira de Medicina Tropical, vol. 49, no. 6, pp. 703-712, 2016.
[2] R. M. Donlan, "Role of biofilms in antimicrobial resistance," ASAIO Journal, vol. 46, no. 6, pp. S47-S52, 2000.

[3] M. Jamal, W. Ahmad, S. Andleeb et al., "Bacterial biofilm and associated infections," Journal of the Chinese Medical Association, vol. 81, no. 1, pp. 7-11, 2018.

[4] R. M. Donlan, "Biofilm formation: a clinically relevant microbiological process," Clinical Infectious Diseases, vol. 33, no. 8, pp. 1387-1392, 2001.

[5] R. Wolcott, J. W. Costerton, D. Raoult, and S. J. Cutler, "The polymicrobial nature of biofilm infection," Clinical Microbiology and Infection, vol. 19, no. 2, pp. 107-112, 2013.

[6] B. Sasirekha, D. Megha, M. S. Chandra, and R. Soujanya, "Study on effect of different plant extracts on microbial biofilms," Asian Journal of Biotechnology, vol. 7, no. 1, pp. 1-12, 2014.

[7] J.-F. Hu, E. Garo, M. G. Goering et al., "Bacterial biofilm inhibitors from Diospyrosdendo," Journal of Natural Products, vol. 69, no. 1, pp. 118-120, 2006.

[8] S. M. Bhola, F. M. Alabbas, R. Bhola et al., "Neem extract as an inhibitor for biocorrosion influenced by sulfate reducing bacteria: a preliminary investigation," Engineering Failure Analysis, vol. 36, pp. 92-103, 2014.

[9] R. M. Gyasi, A. A. Poku, S. Boateng et al., "Integration for coexistence? Implementation of intercultural health care policy in Ghana from the perspective of service users and providers," Journal of Integrative Medicine, vol. 15, no. 1, pp. 44-55, 2017.

[10] M. B. Coyle, Manual of Antimicrobial Susceptibility Testing, American Society for Microbiology, Washington, DC, USA, 2005.

[11] S. Stepanović, D. Vuković, V. Hola et al., "Quantification of biofilm in microtiter plates: overview of testing conditions and practical recommendations for assessment of biofilm production by Staphylococci," APMIS: Acta Pathologica, Microbiologica, Et Immunologica Scandinavica, vol. 115, no. 8, pp. 891-899, 2007.

[12] J. H. Jorgensen and J. D. Turnidge, "Susceptibility test methods: dilution and disk diffusion methods," in Manual of Clinical Microbiology, pp. 1253-1273, American Society of Microbiology, Washington, DC, USA, 11th edition, 2015.

[13] M. M. Amaral, L. R. Coelho, R. P. Flores et al., "The predominant variant of the Brazilian epidemic clonal complex of methicillin-resistant Staphylococcus aureus has an enhanced ability to produce biofilm and to adhere to and invade airway epithelial cells," The Journal of Infectious Diseases, vol. 192, no. 5, pp. 801-810, 2005.

[14] H. Magesh, A. Kumar, A. Alam et al., "Identification of natural compounds which inhibit biofilm formation in clinical isolates of Klebsiella pneumoniae," Indian Journal of Experimental Biology, vol. 51, no. 9, pp. 764-772, 2013.

[15] C. O. Nnadi, N. J. Nwodo, M. Kaiser, R. Brun, and T. J. Schmidt, "Steroid alkaloids from Holarrhena africana with strong activity against Trypanosoma brucei rhodesiense," Molecules, vol. 22, no. 7, p. 1129, 2017.

[16] M. Janot, P. Devissaguet, Q. Khuong-Huu, and R. Goutarel, "Steroid alkaloids. LXVI. New alkaloids from the husks of Holarrhena floribunda (G. Don) Dur. and Schinz: holarrheline, holadienine, holaromine and holaline," Annales Pharmaceutiques Francaises, vol. 25, no. 11, pp. 733-748, 1967.

[17] K. Appiah, C. Oppong, H. Mardani et al., "Medicinal plants used in the Ejisu-Juaben municipality, Southern Ghana: an ethnobotanical study," Medicines, vol. 6, no. 1, 2019. 
[18] N. K. Archer, M. J. Mazaitis, J. W. Costerton, J. G. Leid, M. E. Powers, and M. E. Shirtliff, "Staphylococcus aureus biofilms: properties, regulation and roles in human disease," Virulence, vol. 2, no. 5, pp. 445-459, 2011.

[19] A. M. Lannang, S. Anjum, J. G. Tangmouo, K. Krohn, and M. I. Choudhary, "Conessine isolated from Holarrhena floribunda," Acta Crystallographica Section E: Structure Reports Online, vol. 63, no. 11, 4398 pages, 2007.

[20] H. Burkill, The useful plants of West Africa, Families A-R, Vol. 1, Royal Botanical Gardens, Kew, UK, 1985.

[21] H. Ahmed, "A phyto pharmacological review on a medicinal plant: Holarrhena floribunda," Journal of Medicinal Plants Studies, vol. 5, no. 6, pp. 26-29, 2017.

[22] K. Patrice, P. B. Véronique, L. David, and E. François-Xavier, "Antibacterial activities of the extracts and conessine from Holarrhena floribunda G. Don. (Apocynaceae)," African Journal of Traditional, Complementary, and Alternative Medicines, vol. 4, no. 3, pp. 352-356, 2007.

[23] A. Yemoa, J. Gbenou, D. Affolabi et al., "Beninese medicinal plants as a source of antimycobacterial agents: bioguided fractionation and in vitro activity of alkaloids isolated from Holarrhena floribunda used in traditional treatment of buruli ulcer," BioMed Research International, vol. 2015, Article ID 835767, 5 pages, 2015.

[24] Y. P. Hoekou, T. Tchacondo, S. D. Karou et al., “Therapeutic potentials of ethanolic extract of leaves of Holarrhena floribunda (G. Don) Dur. and schinz (apocynaceae)," African Journal of Traditional, Complementary and Alternative Medicines, vol. 14, no. 2, pp. 227-233, 2017.

[25] P. M. Abreu, E. S. Martins, O. Kayser et al., "Antimicrobial, antitumor and antileishmania screening of medicinal plants from Guinea-Bissau," Phytomedicine, vol. 6, no. 3, pp. 187-195, 1999.

[26] B. Odusina and S. Ibrahim, "Phytochemical screening, antimicrobial activities and quantity estimates of bioactive compounds in extracts of Holarrhea floribunda," Journal of Chemical Society of Nigeria, vol. 43, no. 3, 2018.

[27] M. Janot, A. Cave, and R. Goutarel, "Sterol alkaloids. III. Togholamine, holaphyllamine, and holaphylline, new alkaloids from the leaves of Holarrhena floribunda," Bulletin de la Société Chimique de France, pp. 896-900, 1959.

[28] M. Janot, A. Cave, and R. Goutarel, "Steroid alkaloids. Holaphyllamine and holamine, alkaloids from Holarrhena floribunda (G. Don) Dur. and Schinz," Comptes rendus hebdomadaires des seances de l'Academie des sciences, vol. 251, pp. 559-561, 1960.

[29] J. A. Badmus, O. E. Ekpo, F. Rautenbach, J. L. Marnewick, A. A. Hussein, and D. C. Hiss, "Isolation and antioxidant activity of flavonoids from Holarrhena floribunda (G. don) leaves," Acta Biochimica Polonica, vol. 63, no. 2, pp. 353-358, 2016.

[30] A. Loukaci, O. Kayser, K.-U. Bindseil, K. Siems, J. Frevert, and P. M. Abreu, "New trichothecenes isolated from Holarrhena floribunda," Journal of Natural Products, vol. 63, no. 1, pp. 52-56, 2000.

[31] J. Fotie, D. S. Bohle, M. L. Leimanis, E. Georges, G. Rukunga, and A. E. Nkengfack, "Lupeol long-chain fatty acid esters with antimalarial activity from Holarrhena floribunda," Journal of Natural Products, vol. 69, no. 1, pp. 62-67, 2006.

[32] D. Kavitha, P. Shilpa, and S. N. Devaraj, "Antibacterial and antidiarrhoeal effects of alkaloids of Holarrhena antidysenterica Wall," Indian Journal of Experimental Biology, vol. 42, pp. 589-594, 2004.
[33] A. Chakraborty and A. H. Brantner, "Antibacterial steroid alkaloids from the stem bark of Holarrhena pubescens," Journal of Ethnopharmacology, vol. 68, no. 1-3, pp. 339-344, 1999.

[34] B. S. Siddiqui, S. T. Ali, G. H. Rizwani, S. Begum, S. Tauseef, and A. Ahmad, "Antimicrobial activity of the methanolic bark extract of Holarrhena pubescens (Buch. Ham), its fractions and the pure compound conessine," Natural Product Research, vol. 26, no. 11, pp. 987-992, 2011.

[35] S. Chusri, P. Na-Phatthalung, T. Siriyong, S. Paosen, and S. P. Voravuthikunchai, "Holarrhena antidysenterica as a resistance modifying agent against Acinetobacter baumannii: its effects on bacterial outer membrane permeability and efflux pumps," Microbiological Research, vol. 169, no. 5-6, pp. 417-424, 2014.

[36] T. Siriyong, S. Chusri, P. Srimanote, V. Tipmanee, and S. P. Voravuthikunchai, "Holarrhena antidysenterica extract and its steroidal alkaloid, conessine, as resistance-modifying agents against extensively drug-resistant Acinetobacter baumannii," Microbial Drug Resistance, vol. 22, no. 4, pp. 273-282, 2016.

[37] T. Siriyong, S. P. Voravuthikunchai, and P. J. Coote, "Steroidal alkaloids and conessine from the medicinal plant Holarrhena antidysenterica restore antibiotic efficacy in a Galleria mellonella model of multidrug-resistant Pseudomonas aeruginosa infection," BMC Complementary and Alternative Medicine, vol. 18, no. 1, p. 285, 2018.

[38] T. Siriyong, P. Srimanote, S. Chusri et al., "Conessine as a novel inhibitor of multidrug efflux pump systems in Pseudomonas aeruginosa," BMC Complementary and Alternative Medicine, vol. 17, no. 1, p. 405, 2017.

[39] A. Reza, J. M. Sutton, and K. M. Rahman, "Effectiveness of efflux pump inhibitors as biofilm disruptors and resistance breakers in gram-negative (ESKAPEE) bacteria," Antibiotics, vol. 8, no. 4, 229 pages, 2019.

[40] Z. Li-Na, G. Xiao-Lei, D. Ting-Ting, G. Hui-Yuan, and S. BoHang, "Antibacterial steroidal alkaloids from Holarrhena antidysenteriaca," Chinese Journal of Natural Medicines, vol. 15, no. 7, pp. 540-545, 2017.

[41] A. Tanwar, R. Chawla, A. S. Chakotiya et al., "Effect of Holarrhena antidysentrica (Ha) and Andrographis paniculata (Ap) on the biofilm formation and cell membrane integrity of opportunistic pathogen Salmonella typhimurium," Microbial Pathogenesis, vol. 101, pp. 76-82, 2016. 